\title{
Scalable Consensus Filtering for Uncertain Systems over Sensor Networks with Round-Robin Protocol
}

\author{
Fei Han, Zidong Wang, Guanrong Chen and Hongli Dong*
}

\begin{abstract}
This paper is concerned with the scalable distributed $H_{\infty}$-consensus filtering problem for a class of discrete time-varying systems over sensor networks with the Round-Robin protocol. The challenge comes from the fact that the time-varying parameters of the network are subject to randomly occurring norm-bounded uncertainties and the measurement outputs of the sensor nodes are saturated due to the sector nonlinearities. For preventing data collisions and saving energy, the Round-Robin protocol determines which neighboring node can access the shared network for information transmission at each time step. An $H_{\infty}$ performance index is proposed to characterize the disturbance attenuation level of the resulting filtering error dynamics. By stochastic analysis in combination with the recursive matrix inequality approach, a distributed filtering algorithm is developed for each individual sensor node to ensure the pre-specified estimation performance. Finally, an illustrative simulation example is shown to verify the effectiveness and applicability of the theoretical results.
\end{abstract}

\section{Index Terms}

Distributed filtering; dissipation matrix; $H_{\infty}$-consensus; Round-Robin protocol; scalability; sensor network

\section{INTRODUCTION}

The past few decades have seen a popularity surge with sensor networks (SNs) for their wide range of applications in engineering practice including environmental monitoring, fire detection, target tracking and vehicular ad-hoc networks [5], [24], [38], [40]. One of the key SN-related research topics is distributed filtering that has proven to possess advantages in simplicity, efficiency, robustness and flexibility over the conventional centralized algorithms [10], [21], [27], [41]. Distributed filtering methodologies have attracted a great deal of attention and some widely investigated schemes include distributed Kalman filtering [3], [9], [15], [19], [28], [44], distributed $H_{\infty}$ filtering [4], [11], [14], [33], [34], [36], [37], [39], distributed fusion filtering [2], [30], [31] and distributed set-membership filtering [22], [26].

This work was supported in part by the National Natural Science Foundation of China under Grants 61873058, 61873148, 61933007 and 62073070, the China Postdoctoral Science Foundation under Grants 2017M621242 and 2020T130092, the PetroChina Innovation Foundation under Grant 2018D-5007-0302, the Natural Science Foundation of Heilongjiang Province of China under Grant F2018005, the Fundamental Research Funds for Provincial Undergraduate Universities of Heilongjiang Province of China under Grants 2018QNL-05 and KYCXTD201802, and the Alexander von Humboldt Foundation of Germany.

F. Han and H. Dong are with the Artificial Intelligence Energy Research Institute, Northeast Petroleum University, Daqing, 163318, China. They are also with the Heilongjiang Provincial Key Laboratory of Networking and Intelligent Control, Northeast Petroleum University, Daqing, 163318, China.

Z. Wang is with the Department of Computer Science, Brunel University London, Uxbridge, Middlesex, UB8 3PH, UK.

G. Chen is with the Department of Electrical Engineering, City University of Hong Kong, Hong Kong, China.

${ }^{*}$ Corresponding author. E-mail: shiningdhl@vip.126.com. 
An SN is comprised of a large number of sensor nodes for sensing, computing and communicating. In response to the large-scale characteristics of SNs, a desired filtering algorithm should be scalable; that is, the filter gains can be computed separately on each individual node and also is adaptive and flexible to the changes of the network topology. Notably, the scalability issue has attracted growing research interest in the past two years or so [7].

One of the main challenges associated with distributed filtering over sensor networks is the complicated couplings among the large number of nodes. A common technique for tackling such a challenge is to pack the couplings into a single system of larger scale that could then be dealt with by certain existing methods. However, in such an augmented framework, the desired filter gains can only be centrally calculated in an off-line manner by using information collected from the entire network. This type of centralized technique is impractical because of the lack of the desirable scalability. In view of this, a novel idea of local design was proposed and implemented in [11]-[13], [33], where each node can only use information received from its neighboring nodes. Since such local design method is helpful for designing scalable distributed filters, it was quickly recognized and implemented in different scenarios. For instance, a distributed robust estimation problem was investigated in [33] for continuoustime systems, and a similar problem was studied in [11] for systems with stochastic nonlinearities and multiple missing measurements. In this paper, with the help from such local design methodology, the scalability of the distributed filtering problem is studied for uncertain stochastic sensor networks.

As is well known, network protocols play a predominant role in communication systems for regulating data transmission to avoid congestion and collision. It is of significance to examine the impact of protocols on various filtering issues especially in the context of sensor networks with respect to their topologies. To date, much effort has been devoted to the investigation of filtering and control problems subject to different types of protocols such as Round-Robin protocol (RRP) [6], [23], [34], [35], [48], Weighted Try-Once-Discard protocol (WTODP) [6], and stochastic communication protocol (SCP) [17], [44]. In particular, the well-known Round-Robin protocol (also named as time-division multiple access protocol or token ring protocol) has been extensively used in various filtering/estimation problems. Under this protocol, each node is assigned equitable access to the shared communication channel according to a fixed circular order allotted by the scheduler. More specifically, a moving horizon estimation problem was discussed in [47] for a class of networked time-delay systems under the RRP, which was adopted to determine the transmission order of sensor nodes. A distributed robust estimation algorithm was proposed in [34], where the RRP was utilized to schedule data transmission on a network. Very recently, a so-called full-information state estimator was designed in [48] for a class of linear time-varying systems with the RRP, where an effective full-information estimator design framework has been proposed for time-varying systems. It is worth mentioning that the RRP is perhaps the most frequently applied protocol for its easy deployability in engineering practice.

This paper investigates the scalable distributed filtering problem for SNs with RRP. The task faces with two challenges. On one hand, saturation phenomenon has long been recognized to be inevitably popular in engineering systems because of the limited measuring capability of sensor and actuator devices. Note that sensor saturation could result in nonlinear characteristics which, in turn, would lead to performance degradation or even instability. Accordingly, considerable effort has been dedicated to advanced filter design strategies in the occurrence of sensor saturations; see, e.g. [25], [26], [43], for some recent publications. On the other hand, in practice, almost all systems contain parameters that are inherently uncertain and variable owing primarily to the evolution of the systems or the changes of the environments. Such parameter variations often occur in a random way, especially in networked systems where the network load is typically unpredictable. The randomly occurring parameter uncertainties (ROPUs) 
have recently attracted a great deal of attention; see, e.g. [14], [29]. It should be emphasized that the scalability, as discussed previously, requires local design on each sensor node, which would become substantially challenging due to the existence of the global ROPUs. In the current literature, the distributed filtering problem has not been fully addressed for systems suffering from the ROPUs, where the RRP has not been used for routing.

Summarizing the above discussions, it can be concluded that 1) local design methodology can help with increasing the scalability of the distributed filter; 2) deployment of the RRP could enhance the utilization efficiency given limited network resource; and 3) sensor saturations and randomly occurring parameter uncertainties (ROPUs) are two major factors contributing to the system complexity that should be taken into careful consideration in the design. As such, it is both theoretically important and practically significant to study the distributed filtering problem for uncertain time-varying systems with the RRP, and the corresponding technical questions are identified as follows: 1) how to schedule the neighboring information according to the RRP in a distributed filtering scheme? 2) how to deal with the global norm-bounded uncertainties in the scalable design for distributed consensus filters? 3) how to establish a novel performance index corresponding to the RRP? and 4) how to achieve and further verify the scalability of the distributed filtering algorithm? This paper addresses these questions by a thorough investigation.

In brief, this paper studies the scalable design problem of distributed filters, to be implemented on each node, for systems with ROPUs and saturated measurements with RRP. The main contributions of this paper are highlighted as follows:1) the scalability issue is, for the first time, investigated in the framework of local design for the distributed filtering problem with RRP; 2) inspired by the existing results concerning the RRP and the distributed $H_{\infty}$-consensus filtering, a novel $H_{\infty}$-consensus performance index is proposed that reflects the impact of RRP and also accounts for the disturbance attenuation levels of both filtering errors and consensus errors; 3) different from [14], the randomly occurring norm-bounded uncertainties are dedicatedly handled so as to keep the design locality intact with the distributed filters.

The remaining of this paper is organized as follows. In Section II, the target plant and the distributed filtering problem are formulated. In Section III, the scalable distributed filtering with RRP is designed using the vector dissipation theory. An illustrative example is presented in Section IV to demonstrate the effectiveness and the applicability of the proposed filtering algorithm. Finally, in Section V, conclusions are drawn and a few future research topics are outlined.

Notation. For two column vectors $x, y \in \mathbb{R}^{m}, x \geq \geq y$ (respectively, $x \leq \leq y$ ) means that every element of $x$ is greater than or equal to (respectively, less than or equal to) the corresponding element of $y$. 1 denotes a column vector with every element being 1 . A nonnegative square matrix $W$ is column substochastic if $\mathbf{1}^{T} W \leq \leq \mathbf{1}^{T}$. $l_{2}[0, n-1]$ represents the space of summable vector sequences over $[0, n-1]$. For an $m$-dimensional vector sequence $w_{k} \in l_{2}[0, n-1]$ and a weight matrix $Q_{k} \in \mathbb{R}^{m \times m},\left\|w_{k}\right\|_{Q_{k}}^{2}=w_{k}^{T} Q_{k} w_{k} \cdot \operatorname{diag}\{\ldots\}$ denotes a block diagonal matrix. For an integer $a$ and a positive integer $b, \bmod (a, b)$ represents the unique nonnegative remainder from division of $a$ by $b$.

\section{PROBLEM Formulation}

Consider a sensor network of $N$ nodes with topology represented by a digraph $\mathcal{G}=(\mathcal{V}, \mathcal{E}, \mathscr{A})$ having the set of nodes $\mathcal{V}=\{i \mid i=1,2, \ldots, N\}$ and the set of edges $\mathcal{E}=\{(i, j) \mid(i, j) \in \mathcal{V} \times \mathcal{V}\}$. In the adjacency matrix $\mathscr{A}=\left[a_{i j}\right] \in \mathbb{R}^{N \times N}$, if $(i, j) \in \mathcal{E}$ and $i \neq j$, then $a_{i j}=1$; otherwise, $a_{i j}=0 ; a_{i j}=1$ means that node $j$ can 
provide information to node $i$. Moreover, $p_{i} \triangleq \sum_{j=1}^{N} a_{i j}$ and $q_{i} \triangleq \sum_{j=1}^{N} a_{j i}$ are, respectively, the in-degree and the out-degree of node $i$.

Consider the following discrete-time stochastic system with randomly occurring norm-bounded uncertainties defined on $k \in \mathcal{H} \triangleq\{0,1, \ldots, n-1\}$ :

$$
x_{k+1}=\left(A_{k}+\beta_{k} \Delta A_{k}\right) x_{k}+\left(B_{k}+\beta_{k} \Delta B_{k}\right) w_{k},
$$

with $N$ saturated sensor measurements modeled by

$$
y_{i, k}=\sigma_{i}\left(C_{i, k} x_{k}\right)+D_{i, k} \vartheta_{i, k}, \quad i \in \mathcal{V},
$$

where $x_{k} \in \mathbb{R}^{n_{x}}$ is the state, $y_{i, k} \in \mathbb{R}^{n_{y}}$ is the measurement of node $i, w_{k} \in \mathbb{R}^{n_{w}}$ and $\vartheta_{i, k} \in \mathbb{R}^{n_{\vartheta}}$ are the external disturbances belonging to $l_{2}[0, n-1]$, and $A_{k}, B_{k}, C_{i, k}$ and $D_{i, k}$ are known time-varying matrices with compatible dimensions.

Assume that the time-varying parameter uncertainties $\Delta A_{k}$ and $\Delta B_{k}$ satisfy

$$
\left[\Delta A_{k} \Delta B_{k}\right]=M_{k} F_{k}\left[N_{1 k} N_{2 k}\right]
$$

where $M_{k}, N_{1 k}$ and $N_{2 k}$ are known time-varying matrices with appropriate dimensions, and $F_{k}$ is an unknown time-varying matrix satisfying the constraint

$$
F_{k} F_{k}^{T} \leq I, k \in \mathcal{H}
$$

The random variable $\beta_{k}$ is a Bernoulli distributed sequence taking values either 0 or 1 with the following probabilities:

$$
\operatorname{Prob}\left\{\beta_{k}=0\right\}=1-\bar{\beta}_{k}, \quad \operatorname{Prob}\left\{\beta_{k}=1\right\}=\bar{\beta}_{k},
$$

where $\bar{\beta}_{k}$ is a known constant belonging to $[0,1]$.

The saturation function $\sigma_{i}(\cdot): \mathbb{R}^{n_{y}} \rightarrow \mathbb{R}^{n_{y}}$ is defined as

$$
\sigma_{i}(z)=\left[\begin{array}{llll}
\sigma_{i 1}^{T}\left(z_{1}\right) & \sigma_{i 2}^{T}\left(z_{2}\right) & \cdots & \sigma_{i n_{y}}^{T}\left(z_{n_{y}}\right)
\end{array}\right]^{T}
$$

with $\sigma_{i}^{T}\left(z_{i}\right)=\operatorname{sign}\left(z_{i}\right) \min \left\{z_{i, \max },\left|z_{i}\right|\right\}$, where $z_{i}$ is the $i$ th element of the vector $z$ and $z_{i, \max }$ is the saturation level of $z_{i}$.

Assuming that there exist diagonal matrices $H_{i 1}$ and $H_{i 2}$ such that $0 \leq H_{i 1}<I \leq H_{i 2}<2 I$, the saturation function $\sigma_{i}\left(C_{i, k} x_{k}\right)$ in (2) can be decomposed into a linear part and a nonlinear part as

$$
\sigma_{i}\left(C_{i, k} x_{k}\right)=\bar{H}_{i} C_{i, k} x_{k}+\Phi_{i}\left(C_{i, k} x_{k}\right)
$$

where $\Phi_{i}\left(C_{i, k} x_{k}\right)$ is a nonlinear vector-valued function satisfying

$$
\Phi_{i}^{T}\left(C_{i, k} x_{k}\right) \Phi_{i}\left(C_{i, k} x_{k}\right) \leq x_{k}^{T} C_{i, k}^{T} H_{i}^{T} H_{i} C_{i, k} x_{k}
$$

with $\bar{H}_{i}=\frac{1}{2}\left(H_{i 1}+H_{i 2}\right)$ and $H_{i}=\frac{1}{2}\left(H_{i 2}-H_{i 1}\right)$.

Considering the limited capacity of the communication channel, the RRP is adopted to schedule the data transmission and make full utilization of the shared network resources. In the neighbor set of node $i$, denoted as $\mathcal{N}_{i}=\left\{j_{i 1}, j_{i 2}, \cdots, j_{i p_{i}}\right\}$, let the first transmission node be $j_{i 1}$. Denote by $\hbar_{i, k}=\bmod \left(k-1, p_{i}\right)+1$ the subscript of the selected neighbor of node $i$ having access to the shared communication network. Thus, $j_{\hbar_{i, k}} \in \mathcal{N}_{i}$. 
In this paper, the following distributed filter is designed for node $i$ :

$$
\hat{x}_{i, k+1}=A_{k} \hat{x}_{i, k}+L_{i, k}\left(y_{i, k}-\bar{H}_{i} C_{i, k} \hat{x}_{i, k}\right)+K_{i j_{\hbar_{i, k}}, k}\left(\hat{x}_{j_{\hbar_{i, k}, k}}-\hat{x}_{i, k}\right),
$$

where $\hat{x}_{i, k}$ and $\hat{x}_{j_{\hbar_{i, k}}, k}$ are, respectively, the estimates of $x_{k}$ by node $i$ and its neighboring node $j_{\hbar_{i, k}} \in \mathcal{N}_{i}$. Here, the filter gains $L_{i, k}$ and $K_{i j_{\hbar_{i, k}}, k}$ are to be determined later.

Assumption 1: If a node has no neighboring node, then it is uniformly observable. If a node has neighboring nodes, then it is uniformly distributed observable under the RRP.

To further simplify the expressions, denote $K_{i j_{\hbar}, k}=K_{i j_{\hbar_{i, k}}, k}$ and $\hat{x}_{j_{\hbar_{i}}, k}=\hat{x}_{j_{\hbar_{i, k}}, k}$. Let $e_{i, k}=x_{k}-\hat{x}_{i, k}$ be the filtering error of node $i$. Then, combining (1), (2), (7) and (9), one has the following filtering error dynamics:

$$
\begin{aligned}
e_{i, k+1}= & \left(A_{k}-L_{i, k} \bar{H}_{i} C_{i, k}-K_{i j_{\hbar_{i}}, k}\right) e_{i, k}-L_{i, k}\left(\Phi_{i}\left(C_{i, k} x_{k}\right)+D_{i, k} v_{i, k}\right)+B_{k} w_{k} \\
& +K_{i j_{\hbar_{i}}, k} e_{j_{\hbar_{i}}, k}+\beta_{k}\left(\Delta B_{k} w_{k}+\Delta A_{k} x_{k}\right) .
\end{aligned}
$$

Denoting $\eta_{i, k}=\left[\begin{array}{ll}x_{k}^{T} & e_{i, k}^{T}\end{array}\right]^{T}, \xi_{i, k}=\left[\begin{array}{ll}w_{k}^{T} & \vartheta_{i, k}^{T}\end{array}\right]^{T}, z_{i, k}=\left[\begin{array}{ll}0 & I\end{array}\right] \eta_{i, k} \triangleq E \eta_{i, k}$ and $\tilde{\beta}_{k}=\beta_{k}-\bar{\beta}_{k}$, one has

$$
\left\{\begin{aligned}
\eta_{i, k+1}= & \left(\mathcal{A}_{i, k}+\bar{\beta}_{k} \Delta \mathcal{A}_{k}\right) \eta_{i, k}+\mathcal{K}_{i j_{\hbar}, k} \eta_{j \hbar_{i}, k}+\tilde{\beta}_{k} \Delta \mathcal{A}_{k} \eta_{i, k}+\mathcal{L}_{i, k} \bar{\Phi}_{i}\left(\mathcal{C}_{i, k} \eta_{i, k}\right) \\
& +\left(\mathcal{B}_{i, k}+\bar{\beta}_{k} \Delta \mathcal{B}_{k}\right) \xi_{i, k}+\tilde{\beta}_{k} \Delta \mathcal{B}_{k} \xi_{i, k} \\
z_{i, k}= & E \eta_{i, k}
\end{aligned}\right.
$$

where

$$
\begin{aligned}
& \mathcal{A}_{i, k}=\operatorname{diag}\left\{A_{k}, A_{k}-L_{i, k} \bar{H}_{i} C_{i, k}-K_{i j_{\hbar}, k}\right\}, \\
& \mathcal{C}_{i, k}=\operatorname{diag}\left\{C_{i, k}, 0\right\}, \quad \mathcal{K}_{i j_{\hbar}, k}=\operatorname{diag}\left\{0, K_{i j_{\hbar}, k}\right\}, \\
& \mathcal{B}_{i, k}=\left[\begin{array}{cc}
B_{k} & 0 \\
B_{k} & -L_{i, k} D_{i, k}
\end{array}\right], \Delta \mathcal{A}_{k}=\left[\begin{array}{cc}
\Delta A_{k} & 0 \\
\Delta A_{k} & 0
\end{array}\right], \\
& \Delta \mathcal{B}_{k}=\left[\begin{array}{cc}
\Delta B_{k} & 0 \\
\Delta B_{k} & 0
\end{array}\right], \quad \mathcal{L}_{i, k}=\left[\begin{array}{cc}
0 & 0 \\
-L_{i, k} & 0
\end{array}\right], \\
& \bar{\Phi}_{i}\left(\mathcal{C}_{i, k} \eta_{i, k}\right)=\left[\begin{array}{ll}
\Phi_{i}^{T}\left(C_{i, k} x_{k}\right) & 0
\end{array}\right]^{T} .
\end{aligned}
$$

Also, (8) can be rewritten as

$$
\bar{\Phi}_{i}^{T}\left(\mathcal{C}_{i, k} \eta_{i, k}\right) \bar{\Phi}_{i}\left(\mathcal{C}_{i, k} \eta_{i, k}\right) \leq \eta_{i, k}^{T} \mathcal{C}_{i, k}^{T} \mathcal{H}_{i}^{T} \mathcal{H}_{i} \mathcal{C}_{i, k} \eta_{i, k}
$$

where $\mathcal{H}_{i}=\operatorname{diag}\left\{H_{i}, 0\right\}$.

Before proceeding further, introduce the following performance index to characterize the disturbance attenuation level of the filtering error dynamics (11) against external disturbances.

Definition 1: Let the disturbance attenuation level $\gamma>0$ and the weighting matrices $\mathcal{U}_{i}, R_{i, k}, Q_{i, k}$ and $\mathcal{T}_{i, k}$ be given. The filtering error system (11) is said to satisfy the $H_{\infty}$-consensus performance constraint over the finite horizon $\mathcal{H}$ with the RRP, if the following inequality holds:

$$
\mathbb{E}\left\{\sum_{k=0}^{n-1} \sum_{i=1}^{N}\left[\left\|z_{j_{\hbar_{i}}, k}-z_{i, k}\right\|_{R_{i, k}}^{2}+\left\|z_{i, k}\right\|_{Q_{i, k}}^{2}\right]\right\} \leq \gamma^{2} \sum_{i=1}^{N}\left(\left\|\eta_{i, 0}\right\|_{\mathcal{U}_{i}}^{2}+\sum_{k=0}^{n-1}\left\|\xi_{i, k}\right\|_{\mathcal{T}_{i, k}}^{2}\right),
$$

where $\mathcal{U}_{i}=\operatorname{diag}\left\{U_{1 i}, U_{2 i}\right\}, \mathcal{T}_{i, k}=\operatorname{diag}\left\{T_{1 i, k}, T_{2 i, k}\right\}$, and $U_{1 i}, U_{2 i}, R_{i, k}, Q_{i, k}, T_{1 i, k}$ and $T_{2 i, k}$ are all known positive definite matrices. 
The main objective in this paper is to determine the filter gains $L_{i, k}$ and $K_{i j_{\hbar}, k}$ such that the filtering error dynamics (11) satisfies the proposed $H_{\infty}$-consensus performance constraint over the finite horizon.

Remark 1: The consensus-based performance index defined in (13) distinguishes itself from those existing in the literature through evaluating the effect from the RRP, which can be confirmed from the subscript $j_{\hbar_{i}}$ of the term $\sum_{k=0}^{n-1} \sum_{i=1}^{N}\left\|z_{j_{\hbar_{i}}, k}-z_{i, k}\right\|_{R_{i, k}}^{2}$. Accordingly, in case that the RRP is not considered, the proposed performance index is degenerated to the one in [34], which means that the performance measure here is more general. Furthermore, from the engineering point of view, the proposed performance cost is essentially different from the existing ones for the following four reasons: 1) it accounts for the dynamical change of neighboring sensors; 2) the introduction of the weighted matrices $Q_{i, k}$ and $R_{i, k}$ provides a desirable tradeoff between the consensus and the filtering accuracy; 3 ) the performance cost enables one to only investigate the impacts on the filtering accuracy by setting $Q_{i, k}=I$ and $R_{i, k}=0$ or the consensus with $Q_{i, k}=0$ and $R_{i, k}=I$; and 4) with the new cost function, the selected vector-type storage functions and vector-type supply rate functions, to be discussed later, depend on a time-varying subset of the neighbors of sensor $i$.

\section{MAIN RESUlTS}

In this section, several sufficient conditions are obtained for the filtering error dynamics (11) to satisfy the $H_{\infty}$-consensus performance criterion (13).

For convenience, denote

$$
S_{i}\left(z_{i, k}, z_{j_{\hbar_{i}}, k}, \xi_{i, k}\right) \triangleq \gamma^{2}\left\|\xi_{i, k}\right\|_{\mathcal{T}_{i, k}}^{2}-\left\|z_{i, k}\right\|_{Q_{i, k}}^{2}-\left\|z_{j_{\hbar_{i}}, k}-z_{i, k}\right\|_{R_{i, k}}^{2} .
$$

Definition 2: [16] The filtering error system (11) is said to be stochastically vector-dissipative over the finite horizon $\mathcal{H}$ with respect to the vector of supply rate functions $\mathbf{S}\left(\eta_{k}, \xi_{k}\right)=\left[S_{1}\left(z_{1, k}, z_{j_{\hbar_{1}}, k}, \xi_{1, k}\right), \ldots, S_{N}\left(z_{N, k}, z_{j_{\hbar_{N}}, k}, \xi_{N, k}\right)\right]^{T}$, if there exists a vector of nonnegative definite storage functions $\mathbf{V}\left(\eta_{k}\right) \triangleq\left[V_{1}\left(\eta_{1, k}\right), \ldots, V_{N}\left(\eta_{N, k}\right)\right]^{T}$ (with $\left.\mathbf{V}(0)=0\right)$ and a sequence of nonsingular column substochastic dissipation matrices $W_{k} \in \mathbb{R}^{N \times N}$ such that the following vector dissipation inequality is satisfied for all $k \in \mathcal{H}$ :

$$
\mathbb{E}\left\{\mathbf{V}\left(\eta_{k+1}\right)\right\} \leq \leq W_{k} \mathbb{E}\left\{\mathbf{V}\left(\eta_{k}\right)\right\}+\mathbb{E}\left\{\mathbf{S}\left(z_{k}, \xi_{k}\right)\right\}
$$

Next, some local conditions are established for each node such that the filtering error dynamics (11) is stochastically vector-dissipative over the finite horizon $\mathcal{H}$. Define an interval function $\mathscr{I}_{q_{i}}$ of the out-degree $q_{i}$ as follows:

$$
\mathscr{I}_{q_{i}}= \begin{cases}\left(0, \frac{1+q_{i}}{2 q_{i}}\right), & \text { if } q_{i} \neq 0 \\ (0,1], & \text { if } q_{i}=0\end{cases}
$$

Theorem 1: Let the disturbance attenuation level $\gamma>0$, the scalar sequence $\alpha_{i, k} \in \mathscr{I}_{q_{i}}$, the matrices $R_{i, k}$, $Q_{i, k}, \mathcal{U}_{i}, \mathcal{T}_{i, k}$, and the filter gain sequences $L_{i, k}$ and $K_{i j, k}$ be given. System (11) is stochastically vector-dissipative over the finite horizon $\mathcal{H}$ with respect to the vector supply rate $\mathbf{S}\left(z_{k}, \xi_{k}\right)$ and also satisfies the $H_{\infty}$-consensus performance criterion (13), if there exist a sequence of positive scalars $\lambda_{i, k}$ and a vector of storage functions $\mathbf{V}\left(\eta_{k}\right)$ in the form $V_{i}\left(\eta_{i, k}\right)=\eta_{i, k}^{T} \mathcal{P}_{i, k} \eta_{i, k}$, where $\left\{\mathcal{P}_{i, k}\right\}_{k \in \mathcal{H} \cup\{n\}}$ is a sequence of positive definite matrices with initial conditions $\mathcal{P}_{i, 0} \leq \gamma^{2} \mathcal{U}_{i}$, such that the following conditions hold for all $k \in \mathcal{H}, i \in \mathcal{V}$ :

$$
\Xi_{i j_{\hbar}, k}=\left[\begin{array}{cccc}
\Xi_{i j_{\hbar}, k}^{11} & * & * & * \\
\Xi_{i j_{\hbar}, k}^{21} & \Xi_{i j_{\hbar}, k}^{22} & * & * \\
\Xi_{i j_{\hbar}, k}^{31} & \Xi_{i j_{\hbar}, k}^{32} & \Xi_{i j_{\hbar}, k}^{33} & * \\
\Xi_{i j_{\hbar}, k}^{41} & \Xi_{i j_{\hbar}, k}^{42} & \Xi_{i j_{\hbar}, k}^{43} & \Xi_{i j_{\hbar}, k}^{44}
\end{array}\right] \leq 0,
$$


where

$$
\begin{aligned}
\Xi_{i j_{\hbar}, k}^{11}= & \left(\mathcal{A}_{i, k}+\bar{\beta}_{k} \Delta \mathcal{A}_{k}\right)^{T} \mathcal{P}_{i, k+1}\left(\mathcal{A}_{i, k}+\bar{\beta}_{k} \Delta \mathcal{A}_{k}\right)-\theta_{i, k} \mathcal{P}_{i, k}+\varphi_{k} \Delta \mathcal{A}_{k}^{T} \mathcal{P}_{i, k+1} \Delta \mathcal{A}_{k} \\
& +E^{T} R_{i, k} E+E^{T} Q_{i, k} E+\lambda_{i, k} \mathcal{C}_{i, k}^{T} \mathcal{H}_{i}^{T} \mathcal{H}_{i} \mathcal{C}_{i, k}, \\
\Xi_{i j_{\hbar}, k}^{21}= & \mathcal{K}_{i j_{\hbar}, k}^{T} \mathcal{P}_{i, k+1}\left(\mathcal{A}_{i, k}+\bar{\beta}_{k} \Delta \mathcal{A}_{k}\right)-E^{T} R_{i, k} E \\
\Xi_{i j_{\hbar}, k}^{22}= & \mathcal{K}_{i j_{\hbar}, k}^{T} \mathcal{P}_{i, k+1} \mathcal{K}_{i j_{\hbar}, k}+E^{T} R_{i, k} E-\frac{\alpha_{j_{\hbar_{i}}, k}}{1+q_{j_{\hbar_{i}}}} \mathcal{P}_{j_{\hbar_{i}}, k}, \\
\Xi_{i j_{\hbar}, k}^{31}= & \mathcal{L}_{i, k}^{T} \mathcal{P}_{i, k+1}\left(\mathcal{A}_{i, k}+\bar{\beta}_{k} \Delta \mathcal{A}_{k}\right), \\
\Xi_{i j_{\hbar}, k}^{32}= & \mathcal{L}_{i, k}^{T} \mathcal{P}_{i, k+1} \mathcal{K}_{i j_{\hbar}, k}, \quad \Xi_{i j_{\hbar}, k}^{33}=\mathcal{L}_{i, k}^{T} \mathcal{P}_{i, k+1} \mathcal{L}_{i, k}-\lambda_{i, k} I, \\
\Xi_{i j_{\hbar}, k}^{41}= & \left(\mathcal{B}_{i, k}+\bar{\beta}_{k} \Delta \mathcal{B}_{k}\right)^{T} \mathcal{P}_{i, k+1}\left(\mathcal{A}_{i, k}+\bar{\beta}_{k} \Delta \mathcal{A}_{k}\right)+\varphi_{k} \Delta \mathcal{B}_{k}^{T} \mathcal{P}_{i, k+1} \Delta \mathcal{A}_{k}, \\
\Xi_{i j_{\hbar}, k}^{42}= & \left(\mathcal{B}_{i, k}+\bar{\beta}_{k} \Delta \mathcal{B}_{k}\right)^{T} \mathcal{P}_{i, k+1} \mathcal{K}_{i j_{\hbar}, k}, \quad \Xi_{i j_{\hbar}, k}^{43}=\left(\mathcal{B}_{i, k}+\bar{\beta}_{k} \Delta \mathcal{B}_{k}\right)^{T} \mathcal{P}_{i, k+1} \mathcal{L}_{i, k}, \\
\Xi_{i j_{\hbar}, k}^{44}= & \left(\mathcal{B}_{i, k}+\bar{\beta}_{k} \Delta \mathcal{B}_{k}\right)^{T} \mathcal{P}_{i, k+1}\left(\mathcal{B}_{i, k}+\bar{\beta}_{k} \Delta \mathcal{B}_{k}\right)-\gamma^{2} \mathcal{T}_{i, k}+\varphi_{k} \Delta \mathcal{B}_{k}^{T} \mathcal{P}_{i, k+1} \Delta \mathcal{B}_{k}, \\
\theta_{i, k}= & \frac{1+q_{i}\left(1-\alpha_{i, k}\right)}{1+q_{i}}, \quad \varphi_{k}=\bar{\beta}_{k}-\bar{\beta}_{k}^{2} .
\end{aligned}
$$

Proof: Step 1) Proof of the stochastic vector-dissipativity over the finite horizon $\mathcal{H}$.

First of all, calculate the storage function concerning $i$-th node along the trajectory of system (11) as follows:

$$
\begin{aligned}
& \mathbb{E}\left\{V_{i, k+1} \mid \eta_{i, k}\right\} \\
= & \eta_{i, k+1}^{T} \mathcal{P}_{i, k+1} \eta_{i, k+1} \\
= & \eta_{j_{\hbar_{i}}, k}^{T} \mathcal{K}_{i j_{\hbar}, k}^{T} \mathcal{P}_{i, k+1} \mathcal{K}_{i j_{\hbar}, k} \eta_{j_{\hbar_{i}}, k}+\varphi_{k} \eta_{i, k}^{T} \Delta \mathcal{A}_{k}^{T} \mathcal{P}_{i, k+1} \Delta \mathcal{A}_{k} \eta_{i, k} \\
& +2 \varphi_{k} \xi_{i, k}^{T} \Delta \mathcal{B}_{k}^{T} \mathcal{P}_{i, k+1} \Delta \mathcal{A}_{k} \eta_{i, k}+2 \eta_{j_{\hbar_{i}}, k}^{T} \mathcal{K}_{i j_{\hbar}, k}^{T} \mathcal{P}_{i, k+1} \mathcal{L}_{i, k} \bar{\Phi}_{i}\left(\mathcal{C}_{i, k} \eta_{i, k}\right) \\
& +\bar{\Phi}_{i}^{T}\left(\mathcal{C}_{i, k} \eta_{i, k}\right) \mathcal{L}_{i, k}^{T} \mathcal{P}_{i, k+1} \mathcal{L}_{i, k} \bar{\Phi}_{i}\left(\mathcal{C}_{i, k} \eta_{i, k}\right)+2 \eta_{i, k}^{T}\left(\mathcal{A}_{i, k}+\bar{\beta}_{k} \Delta \mathcal{A}_{k}\right)^{T} \mathcal{P}_{i, k+1} \mathcal{K}_{i j_{k}, k} \eta_{j_{\hbar_{i}}, k} \\
& +2 \xi_{i, k}^{T}\left(\mathcal{B}_{i, k}+\bar{\beta}_{k} \Delta \mathcal{B}_{k}\right)^{T} \mathcal{P}_{i, k+1} \mathcal{K}_{i j_{k}, k} \eta_{j_{\hbar_{i}}, k}+2 \xi_{i, k}^{T}\left(\mathcal{B}_{i, k}+\bar{\beta}_{k} \Delta \mathcal{B}_{k}\right)^{T} \mathcal{P}_{i, k+1} \mathcal{L}_{i, k} \bar{\Phi}_{i}\left(\mathcal{C}_{i, k} \eta_{i, k}\right) \\
& +2 \eta_{i, k}^{T}\left(\mathcal{A}_{i, k}+\bar{\beta}_{k} \Delta \mathcal{A}_{k}\right)^{T} \mathcal{P}_{i, k+1} \mathcal{L}_{i, k} \bar{\Phi}_{i}\left(\mathcal{C}_{i, k} \eta_{i, k}\right)+\eta_{i, k}^{T}\left(\mathcal{A}_{i, k}+\bar{\beta}_{k} \Delta \mathcal{A}_{k}\right)^{T} \mathcal{P}_{i, k+1}\left(\mathcal{A}_{i, k}+\bar{\beta}_{k} \Delta \mathcal{A}_{k}\right) \eta_{i, k} \\
& +2 \xi_{i, k}^{T}\left(\mathcal{B}_{i, k}+\bar{\beta}_{k} \Delta \mathcal{B}_{k}\right)^{T} \mathcal{P}_{i, k+1}\left(\mathcal{A}_{i, k}+\bar{\beta}_{k} \Delta \mathcal{A}_{k}\right) \eta_{i, k}+\xi_{i, k}^{T}\left(\mathcal{B}_{i, k}+\bar{\beta}_{k} \Delta \mathcal{B}_{k}\right)^{T} \mathcal{P}_{i, k+1}\left(\mathcal{B}_{i, k}+\bar{\beta}_{k} \Delta \mathcal{B}_{k}\right) \xi_{i, k} \\
& +\xi_{i, k}\left(\varphi_{k} \Delta \mathcal{B}_{k}^{T} \mathcal{P}_{i, k+1} \Delta \mathcal{B}_{k}-\gamma^{2} \mathcal{T}_{i, k}\right) \xi_{i, k}+\gamma^{2} \xi_{i, k}^{T} \mathcal{T}_{i, k} \xi_{i, k} \\
\triangleq & \zeta_{i j_{\hbar}, k}^{T} \Omega_{i j_{n}, k} \zeta_{i j_{\hbar}, k}+\gamma^{2} \xi_{i, k}^{T} \mathcal{T}_{i, k} \xi_{i, k},
\end{aligned}
$$

where

$$
\begin{aligned}
& \zeta_{i j_{\hbar}, k}=\left[\begin{array}{llll}
\eta_{i, k}^{T} & \eta_{j_{\hbar_{i}}, k}^{T} & \bar{\Phi}_{i}^{T}\left(\mathcal{C}_{i, k} \eta_{i, k}\right) & \xi_{i, k}^{T}
\end{array}\right]^{T}, \\
& \Omega_{i j_{\hbar}, k}=\left[\begin{array}{cccc}
\Omega_{i j_{\hbar}, k}^{11} & * & * & * \\
\Omega_{i j_{\hbar}, k}^{21} & \Omega_{i j_{\hbar}, k}^{22} & * & * \\
\Omega_{i j_{\hbar}, k}^{31} & \Omega_{i j_{\hbar}, k}^{32} & \Omega_{i j_{\hbar}, k}^{33} & * \\
\Omega_{i j_{\hbar}, k}^{41} & \Omega_{i j_{\hbar}, k}^{42} & \Omega_{i j_{\hbar}, k}^{43} & \Omega_{i j_{\hbar}, k}^{44}
\end{array}\right] \text {, } \\
& \Omega_{i j_{\hbar}, k}^{11}=\left(\mathcal{A}_{i, k}+\bar{\beta}_{k} \Delta \mathcal{A}_{k}\right)^{T} \mathcal{P}_{i, k+1}\left(\mathcal{A}_{i, k}+\bar{\beta}_{k} \Delta \mathcal{A}_{k}\right)+\varphi_{k} \Delta \mathcal{A}_{k}^{T} \mathcal{P}_{i, k+1} \Delta \mathcal{A}_{k} \text {, } \\
& \Omega_{i j_{\hbar}, k}^{21}=\mathcal{K}_{i j_{\hbar}, k}^{T} \mathcal{P}_{i, k+1}\left(\mathcal{A}_{i, k}+\bar{\beta}_{k} \Delta \mathcal{A}_{k}\right), \quad \Omega_{i j_{\hbar}, k}^{22}=\mathcal{K}_{i j_{\hbar}, k}^{T} \mathcal{P}_{i, k+1} \mathcal{K}_{i j_{\hbar}, k}, \\
& \Omega_{i j_{\hbar}, k}^{31}=\mathcal{L}_{i, k}^{T} \mathcal{P}_{i, k+1}\left(\mathcal{A}_{i, k}+\bar{\beta}_{k} \Delta \mathcal{A}_{k}\right), \quad \Omega_{i j_{\hbar}, k}^{32}=\mathcal{L}_{i, k}^{T} \mathcal{P}_{i, k+1} \mathcal{K}_{i j_{\hbar}, k}, \\
& \Omega_{i j_{\hbar}, k}^{41}=\left(\mathcal{B}_{i, k}+\bar{\beta}_{k} \Delta \mathcal{B}_{k}\right)^{T} \mathcal{P}_{i, k+1}\left(\mathcal{A}_{i, k}+\bar{\beta}_{k} \Delta \mathcal{A}_{k}\right)+\varphi_{k} \Delta \mathcal{B}_{k}^{T} \mathcal{P}_{i, k+1} \Delta \mathcal{A}_{k},
\end{aligned}
$$




$$
\begin{aligned}
& \Omega_{i j_{\hbar}, k}^{42}=\left(\mathcal{B}_{i, k}+\bar{\beta}_{k} \Delta \mathcal{B}_{k}\right)^{T} \mathcal{P}_{i, k+1} \mathcal{K}_{i j_{\hbar}, k}, \quad \Omega_{i j_{\hbar}, k}^{33}=\mathcal{L}_{i, k}^{T} \mathcal{P}_{i, k+1} \mathcal{L}_{i, k}, \\
& \Omega_{i j_{\hbar}, k}^{43}=\left(\mathcal{B}_{i, k}+\bar{\beta}_{k} \Delta \mathcal{B}_{k}\right)^{T} \mathcal{P}_{i, k+1} \mathcal{L}_{i, k}, \\
& \Omega_{i j_{\hbar}, k}^{44}=\left(\mathcal{B}_{i, k}+\bar{\beta}_{k} \Delta \mathcal{B}_{k}\right)^{T} \mathcal{P}_{i, k+1}\left(\mathcal{B}_{i, k}+\bar{\beta}_{k} \Delta \mathcal{B}_{k}\right)+\varphi_{k} \Delta \mathcal{B}_{k}^{T} \mathcal{P}_{i, k+1} \Delta \mathcal{B}_{k}-\gamma^{2} \mathcal{T}_{i, k} .
\end{aligned}
$$

Taking into account (12) and (17), one has

$$
\begin{aligned}
& \zeta_{i j_{\hbar}, k}^{T} \Omega_{i j_{\hbar}, k} \zeta_{i j_{\hbar}, k}+\left\|z_{i, k}\right\|_{Q_{i, k}}^{2}+\left\|z_{j_{\hbar_{i}, k}}-z_{i, k}\right\|_{R_{i, k}}^{2}-\frac{\alpha_{j_{\hbar_{i}}, k}}{1+q_{j_{\hbar_{i}}}} \eta_{j_{\hbar_{i}}, k}^{T} \mathcal{P}_{j_{\hbar_{i}}, k} \eta_{j_{\hbar_{i}}, k} \\
& -\theta_{i, k} \eta_{i, k}^{T} \mathcal{P}_{i, k} \eta_{i, k}-\lambda_{i, k}\left(\bar{\Phi}_{i}^{T}\left(\mathcal{C}_{i, k} \eta_{i, k}\right) \bar{\Phi}_{i}\left(\mathcal{C}_{i, k} \eta_{i, k}\right)-\eta_{i, k}^{T} \mathcal{C}_{i, k}^{T} \mathcal{H}_{i}^{T} \mathcal{H}_{i} \mathcal{C}_{i, k} \eta_{i, k}\right) \\
= & \zeta_{i j_{\hbar}, k}^{T} \Xi_{i j_{\hbar}, k} \zeta_{i j_{\hbar}, k} \leq 0 .
\end{aligned}
$$

On the other hand, it follows from (17) that

$$
\begin{aligned}
& \mathbb{E}\left\{V_{i, k+1} \mid \eta_{i, k}\right\} \\
\leq & \zeta_{i j_{\hbar}, k}^{T} \Omega_{i j_{\hbar}, k} \zeta_{i j_{\hbar}, k}+\gamma^{2} \xi_{i, k}^{T} \mathcal{T}_{i, k} \xi_{i, k}-\lambda_{i, k}\left(\bar{\Phi}_{i}^{T}\left(\mathcal{C}_{i, k} \eta_{i, k}\right) \bar{\Phi}_{i}\left(\mathcal{C}_{i, k} \eta_{i, k}\right)-\eta_{i, k}^{T} \mathcal{C}_{i, k}^{T} \mathcal{H}_{i}^{T} \mathcal{H}_{i} \mathcal{C}_{i, k} \eta_{i, k}\right) \\
\leq & \frac{\alpha_{j_{\hbar_{i}}, k}}{1+q_{j_{\hbar_{i}}}} \eta_{j_{\hbar_{i}}, k}^{T} \mathcal{P}_{j_{\hbar_{i}}, k} \eta_{j_{\hbar_{i}}, k}+\theta_{i, k} \eta_{i, k}^{T} \mathcal{P}_{i, k} \eta_{i, k}+S_{i}\left(z_{i, k}, z_{j_{\hbar_{i}}, k}, \xi_{i, k}\right) \\
= & {\left[0, \ldots, \theta_{i, k}, \ldots, \frac{\alpha_{j_{\hbar_{i}}, k}}{1+q_{j_{\hbar_{i}}}}, \ldots, 0\right] \mathbf{V}\left(\eta_{k}\right)+S_{i}\left(z_{i, k}, z_{j_{\hbar_{i}}, k}, \xi_{i, k}\right) . }
\end{aligned}
$$

Then, defining a new matrix $W_{k}$ with the $i$-th row being $\left[0, \ldots, \theta_{i, k}, \ldots, \frac{\alpha_{j_{\hbar_{i}}, k}}{1+q_{j_{\hbar_{i}}}}, \ldots, 0\right]$, it follows from [11] that $W_{k}$ is the desired dissipation matrix. Consequently,

$$
\mathbb{E}\left\{V_{i, k+1} \mid \eta_{i, k}\right\} \leq\left[W_{k} \mathbf{V}\left(\eta_{k}\right)\right]_{i}+S_{i}\left(z_{i, k}, z_{j_{\hbar_{i}}, k}, \xi_{i, k}\right),
$$

where $\left[W_{k} \mathbf{V}\left(\eta_{k}\right)\right]_{i}$ is the $i$-th element of vector $W_{k} \mathbf{V}\left(\eta_{k}\right)$.

By using properties of the conditional expectation, the following inequality can be obtained:

$$
\mathbb{E}\left\{V_{i, k+1}\right\} \leq \mathbb{E}\left\{\left[W_{k} \mathbf{V}\left(\eta_{k}\right)\right]_{i}\right\}+\mathbb{E}\left\{S_{i}\left(z_{i, k}, z_{j_{\hbar_{i}}, k}, \xi_{i, k}\right)\right\},
$$

which indicates that

$$
\mathbb{E}\left\{\mathbf{V}\left(\eta_{k+1}\right)\right\} \leq \leq W_{k} \mathbb{E}\left\{\mathbf{V}\left(\eta_{k}\right)\right\}+\mathbb{E}\left\{\mathbf{S}\left(z_{k}, \xi_{k}\right)\right\}
$$

Therefore, by Definition 2, the filtering error system (11) is stochastically vector-dissipative.

Step 2) Proof of the guaranteed $H_{\infty}$-consensus performance index.

Left-multiplying $\mathbf{1}^{T}$ to both sides of (19) yields

$$
\mathbf{1}^{T} \mathbb{E}\left\{\mathbf{V}\left(\eta_{k+1}\right)\right\} \leq \mathbf{1}^{T} \mathbb{E}\left\{\mathbf{S}\left(z_{k}, \xi_{k}\right)\right\}+\mathbf{1}^{T} W_{k} \mathbb{E}\left\{\mathbf{V}\left(\eta_{k}\right)\right\} .
$$

Denote $v\left(\eta_{k}\right) \triangleq \mathbf{1}^{T} \mathbb{E}\left\{\mathbf{V}\left(\eta_{k}\right)\right\}$. From $S_{i}\left(z_{i, k}, z_{j_{\hbar_{i}}, k}, \xi_{i, k}\right)$, it follows that

$$
\sum_{i=1}^{N} \mathbb{E}\left\{\left\|z_{i, k}\right\|_{Q_{i, k}}^{2}+\left\|z_{j_{\hbar_{i}}, k}-z_{i, k}\right\|_{R_{i, k}}^{2}\right\} \leq-v\left(\eta_{k+1}\right)+v\left(\eta_{k}\right)+\gamma^{2} \sum_{i=1}^{N}\left\|\xi_{i, k}\right\|_{\mathcal{T}_{i, k}}^{2},
$$

which further implies that

$$
\sum_{k=0}^{n-1} \sum_{i=1}^{N} \mathbb{E}\left\{\left\|z_{i, k}\right\|_{Q_{i, k}}^{2}+\left\|z_{j_{\hbar_{i}}, k}-z_{i, k}\right\|_{R_{i, k}}^{2}\right\} \leq-v\left(\eta_{n}\right)+v\left(\eta_{0}\right)+\gamma^{2} \sum_{k=0}^{n-1} \sum_{i=1}^{N}\left\|\xi_{i, k}\right\|_{\mathcal{T}_{i, k}}^{2} .
$$


Therefore,

$$
\sum_{i=1}^{N} \sum_{k=0}^{n-1} \mathbb{E}\left\{\left\|z_{i, k}\right\|_{Q_{i, k}}^{2}+\left\|z_{j_{\hbar_{i}}, k}-z_{i, k}\right\|_{R_{i, k}}^{2}\right\} \leq \sum_{i=1}^{N}\left(\eta_{i, 0}^{T} \mathcal{P}_{i, 0} \eta_{i, 0}+\gamma^{2} \sum_{k=0}^{n-1}\left\|\xi_{i, k}\right\|_{\mathcal{T}_{i, k}}^{2}\right) .
$$

Taking the initial condition $\mathcal{P}_{i, 0} \leq \gamma^{2} \mathcal{U}_{i}$ into consideration, the $H_{\infty}$-consensus performance constraint (13) is satisfied, which completes the proof.

Remark 2: In the vector dissipation theory proposed in [16], the dissipation matrix has been used to formulate the coupling relationship among the nodes in sensor network. In fact, the construction of the dissipation matrix plays a critical role in achieving the scalable design of the distributed filtering. The novelty of constructing the dissipation matrix in this paper is clarified as follows. Note that, in a representative publication [34], the dissipation matrix $W$ has been constructed as a negative diagonally dominant matrix with the $i$-th row being

$$
\left[\frac{2 \alpha_{1} a_{i 1}}{1+q_{1}}-\hat{\delta}, \cdots,-\frac{2 q_{i} \alpha_{i}}{1+q_{i}}-\hat{\delta}, \cdots, \frac{2 \alpha_{N} a_{i N}}{1+q_{N}}-\hat{\delta}\right]
$$

where $\hat{\delta}$ guarantees that $W$ is negative diagonally dominant and $\mathbf{1}^{T} W \ll-\epsilon \mathbf{1}(\epsilon>0)$. In this paper, a substochastic matrix $W_{k}$ is constructed, with the $i$-th row being

$$
\left[0, \ldots, \frac{1+q_{i}\left(1-\alpha_{i, k}\right)}{1+q_{i}}, \ldots, \frac{\alpha_{j_{\hbar_{i}}, k}}{1+q_{j_{\hbar_{i}}}}, \ldots, 0\right] .
$$

It can be seen that the new structure reflects the distinctive scheduling of the RRP, i.e., every row of this matrix has only two nonzero elements: one is the diagonal element $\frac{1+q_{i}\left(1-\alpha_{i, k}\right)}{1+q_{i}}$, and the other is $\frac{\alpha_{j_{\hbar_{i}}, k}}{1+q_{j_{\hbar_{i}}}}$, where $j_{\hbar_{i}}$ is the chosen neighboring node of $i$ according to the RRP. Moreover, it can be easily found that $\mathbf{1}^{T} W_{k} \ll \mathbf{1}$ and $W_{k}$ is positive diagonally dominant, i.e.,

$$
\frac{1+q_{i}\left(1-\alpha_{i, k}\right)}{1+q_{i}}>\sum_{j=1}^{N} \frac{a_{j i}}{1+q_{i}}=\frac{q_{i}}{1+q_{i}} \geq \sum_{j=1}^{N} W_{j i, k}
$$

since $0<\alpha_{i, k}<\frac{1+q_{i}}{2 q_{i}}$, where $W_{j i, k}$ is the $(j, i)$-th element of $W_{k}$. From the above comparisons, one can conclude that the dissipation matrices constructed in this paper are essentially different from the existing ones and are easier to use.

In the following, the distributed filter gains $L_{i, k}$ and $K_{i j, k}$ are designed based on Theorem 1.

Theorem 2: Given scalars $\alpha_{i, k} \in \mathscr{I}_{q_{i}}$ and matrices $U_{1 i}, U_{2 i}, R_{i, k}, Q_{i, k}$ and $\mathcal{T}_{i, k}$, the filtering error system (11) satisfies the $H_{\infty}$-consensus performance constraint (13) with the pre-specified disturbance attenuation level $\gamma>0$, if there exist matrices $E_{i j_{\hbar}, k}$ and $F_{i, k}$, positive scalars $\lambda_{i, k}$ and $\mu_{i, k}$, and sequences of positive definite matrices $\left\{P_{i, k}^{1}\right\}_{k \in \mathcal{H} \cup\{n\}}$ and $\left\{P_{i, k}^{2}\right\}_{k \in \mathcal{H} \cup\{n\}}$ with initial conditions $P_{i, 0}^{1} \leq \gamma^{2} U_{1 i}$ and $P_{i, 0}^{2} \leq \gamma^{2} U_{2 i}$, such that the following conditions hold for all $k \in \mathcal{H}, i \in \mathcal{V}$ :

$$
\left[\begin{array}{cc}
\Upsilon_{i j_{\hbar}, k}^{a} & * \\
\bar{\Upsilon}_{i j_{\hbar}, k}^{b} & \bar{\Upsilon}_{i j_{\hbar}, k}^{c}
\end{array}\right]<0
$$

where

$$
\bar{\Upsilon}_{i j_{\hbar}, k}^{b}=\left[\begin{array}{cccc}
\overline{\mathcal{A}}_{i, k} & \overline{\mathcal{K}}_{i j_{\hbar}, k} & \overline{\mathcal{L}}_{i, k} & \overline{\mathcal{B}}_{i, k} \\
0 & 0 & 0 & 0 \\
0 & 0 & 0 & 0
\end{array}\right]
$$




$$
\begin{aligned}
& \Upsilon_{i j_{\hbar}, k}^{a}=\left[\begin{array}{cccc}
\Upsilon_{i j_{\hbar}, k}^{11} & * & * & * \\
\Upsilon_{i j_{\hbar}, k}^{21} & \Upsilon_{i j_{\hbar}, k}^{22} & * & * \\
0 & 0 & -\lambda_{i, k} I & * \\
0 & 0 & 0 & \Upsilon_{i j_{\hbar}, k}^{44}
\end{array}\right] \\
& \bar{\Upsilon}_{i j_{\hbar}, k}^{c}=\left[\begin{array}{ccc}
-\mathcal{P}_{i, k+1} & * & * \\
0 & -\mathcal{P}_{i, k+1} & * \\
\bar{\beta}_{k} \overline{\mathcal{M}}_{k}^{T} & \sqrt{\varphi_{k}} \overline{\mathcal{M}}_{k}^{T} & -\frac{\mu_{i, k}}{2} I
\end{array}\right] \text {, } \\
& \Upsilon_{i j_{\hbar}, k}^{11}=E^{T} Q_{i, k} E+E^{T} R_{i, k} E-\theta_{i, k} \mathcal{P}_{i, k}+\mu_{i, k} \mathcal{N}_{1 k}^{T} \mathcal{N}_{1 k}+\lambda_{i, k} \mathcal{C}_{i, k}^{T} \mathcal{H}_{i}^{T} \mathcal{H}_{i} \mathcal{C}_{i, k}, \\
& \Upsilon_{i j_{\hbar}, k}^{21}=-E^{T} R_{i, k} E, \quad \Upsilon_{i j_{\hbar}, k}^{22}=E^{T} R_{i, k} E-\frac{\alpha_{j_{\hbar_{i}}, k}}{1+q_{j_{\hbar_{i}}}} \mathcal{P}_{j_{\hbar_{i}}, k}, \\
& \Upsilon_{i j_{\hbar}, k}^{44}=-\gamma^{2} \mathcal{T}_{i, k}+\mu_{i, k} \mathcal{N}_{2 k}^{T} \mathcal{N}_{2 k} \\
& \overline{\mathcal{A}}_{i, k}=\operatorname{diag}\left\{P_{i, k+1}^{1} A_{k}, P_{i, k+1}^{2} A_{k}-F_{i, k} \bar{H}_{i} C_{i, k}-E_{i j_{k}, k}\right\}, \\
& \overline{\mathcal{K}}_{i j_{\hbar}, k}=\operatorname{diag}\left\{0, E_{i j_{\hbar}, k}\right\}, \quad \mathcal{N}_{1 k}=\left[\begin{array}{ll}
N_{1 k} & N_{1 k}
\end{array}\right], \\
& \mathcal{N}_{2 k}=\left[\begin{array}{ll}
N_{2 k} & N_{2 k}
\end{array}\right], \quad \mathcal{P}_{i, k}=\operatorname{diag}\left\{P_{i, k}^{1}, P_{i, k}^{2}\right\}, \\
& \overline{\mathcal{B}}_{i, k}=\left[\begin{array}{cc}
P_{i, k+1}^{1} B_{k} & 0 \\
P_{i, k+1}^{2} B_{k} & -F_{i, k} D_{i, k}
\end{array}\right], \mathcal{M}_{k}=\left[\begin{array}{c}
M_{k} \\
M_{k}
\end{array}\right], \\
& \overline{\mathcal{L}}_{i, k}=\left[\begin{array}{cc}
0 & 0 \\
-F_{i, k} & 0
\end{array}\right], \quad \overline{\mathcal{M}}_{k}=\left[\begin{array}{c}
P_{i, k+1}^{1} M_{k} \\
P_{i, k+1}^{2} M_{k}
\end{array}\right] .
\end{aligned}
$$

Moreover, the filter gains $L_{i, k}$ and $K_{i j, k}$ are given as follows:

$$
L_{i, k}=\left(P_{i, k+1}^{2}\right)^{-1} F_{i, k}, K_{i j_{\hbar}, k}=\left(P_{i, k+1}^{2}\right)^{-1} E_{i j_{\hbar}, k} .
$$

Proof: First, according to (3), one has

$$
\left[\Delta \mathcal{A}_{k} \Delta \mathcal{B}_{k}\right]=\mathcal{M}_{k} \mathcal{F}_{k}\left[\mathcal{N}_{1 k} \mathcal{N}_{2 k}\right]
$$

where $\mathcal{F}_{k}=\operatorname{diag}\left\{F_{k}, F_{k}\right\}$.

To cope with the uncertainties, (17) is rewritten as

$$
\Xi_{i j_{\hbar}, k}=\Pi_{i j_{\hbar}, k}+\tilde{\mathcal{M}}_{k} \tilde{\mathcal{F}}_{k} \tilde{\mathcal{N}}_{k}+\tilde{\mathcal{N}}_{k}^{T} \tilde{\mathcal{F}}_{k} \tilde{\mathcal{M}}_{k}^{T}
$$

where

$$
\begin{aligned}
& \Pi_{i j_{\hbar}, k}=\left[\begin{array}{cc}
\Pi_{i j_{\hbar}, k}^{a} & * \\
\Pi_{i j_{\hbar}, k}^{b} & \Pi_{i j_{\hbar}, k}^{c}
\end{array}\right] \\
& \Pi_{i j_{\hbar}, k}^{a}=\left[\begin{array}{cccc}
\Pi_{i j_{\hbar}, k}^{11} & * & * & * \\
\Pi_{i j_{\hbar}, k}^{21} & \Pi_{i j_{\hbar}, k}^{22} & * & * \\
0 & 0 & -\lambda_{i, k} I & * \\
0 & 0 & 0 & -\gamma^{2} \mathcal{T}_{i, k}
\end{array}\right] \text {, } \\
& \Pi_{i j_{\hbar}, k}^{b}=\left[\begin{array}{cccc}
\mathcal{A}_{i, k} & \mathcal{K}_{i j_{\hbar}, k} & \mathcal{L}_{i, k} & \mathcal{B}_{i, k} \\
0 & 0 & 0 & 0
\end{array}\right], \quad \Pi_{i j_{\hbar}, k}^{c}=\left[\begin{array}{cc}
-\mathcal{P}_{i, k+1}^{-1} & * \\
0 & -\mathcal{P}_{i, k+1}^{-1}
\end{array}\right] \text {, } \\
& \Pi_{i j_{k}, k}^{11}=E^{T} Q_{i, k} E+E^{T} R_{i, k} E-\theta_{i, k} \mathcal{P}_{i, k}+\lambda_{i, k} \mathcal{C}_{i, k}^{T} \mathcal{H}_{i}^{T} \mathcal{H}_{i} \mathcal{C}_{i, k}, \\
& \Pi_{i j_{\hbar}, k}^{21}=-E^{T} R_{i, k} E, \quad \Pi_{i j_{\hbar}, k}^{22}=E^{T} R_{i, k} E-\frac{\alpha_{j_{\hbar_{i}}, k}}{1+q_{j_{\hbar_{i}}}} \mathcal{P}_{j_{\hbar_{i}}, k},
\end{aligned}
$$




$$
\begin{gathered}
\tilde{\mathcal{M}}_{k}=\left[\begin{array}{cccccc}
0 & * & * & * & * & * \\
0 & 0 & * & * & * & * \\
0 & 0 & 0 & * & * & * \\
0 & 0 & 0 & 0 & * & * \\
\bar{\beta}_{k} \mathcal{M}_{k} & 0 & 0 & \bar{\beta}_{k} \mathcal{M}_{k} & 0 & * \\
\sqrt{\varphi_{k}} \mathcal{M}_{k} & 0 & 0 & \sqrt{\varphi_{k}} \mathcal{M}_{k} & 0 & 0
\end{array}\right], \\
\tilde{\mathcal{F}}_{k}=\operatorname{diag}\left\{\mathcal{F}_{k}, 0,0, \mathcal{F}_{k}, 0,0\right\}, \quad \tilde{\mathcal{N}}_{k}=\operatorname{diag}\left\{\mathcal{N}_{1 k}, 0,0, \mathcal{N}_{2 k}, 0,0\right\} .
\end{gathered}
$$

Since $\mathcal{F}_{k} \mathcal{F}_{k}^{T} \leq I$, it is inferred from S-procedure that inequality (17) holds if and only if there exists a positive constant $\mu_{i, k}$ such that the following inequality holds:

$$
\Pi_{i j_{\hbar}, k}+\mu_{i, k}^{-1} \tilde{\mathcal{M}}_{k} \tilde{\mathcal{M}}_{k}^{T}+\mu_{i, k} \tilde{\mathcal{N}}_{k}^{T} \tilde{\mathcal{N}}_{k}<0,
$$

which, by the Schur Complement Lemma, is equivalent to

$$
\left[\begin{array}{cc}
\Upsilon_{i j_{\hbar}, k}^{a} & * \\
\Upsilon_{i j_{\hbar}, k}^{b} & \Upsilon_{i j_{\hbar}, k}^{c}
\end{array}\right]<0
$$

where

$$
\begin{aligned}
\Upsilon_{i j_{\hbar}, k}^{a}= & {\left[\begin{array}{cccc}
\Upsilon_{i j_{\hbar}, k}^{11} & * & * & * \\
\Upsilon_{i j_{\hbar}, k}^{21} & \Upsilon_{i j_{\hbar}, k}^{22} & * & * \\
0 & 0 & -\lambda_{i, k} I & * \\
0 & 0 & 0 & \Upsilon_{i j_{\hbar}, k}^{44}
\end{array}\right], } \\
\Upsilon_{i j_{\hbar}, k}^{b}= & {\left[\begin{array}{cccc}
\mathcal{A}_{i, k} & \mathcal{K}_{i j_{\hbar}, k} & \mathcal{L}_{i, k} & \mathcal{B}_{i, k} \\
0 & 0 & 0 & 0 \\
0 & 0 & 0 & 0
\end{array}\right], \quad \Upsilon_{i j_{\hbar}, k}^{c}=\left[\begin{array}{ccc}
-\mathcal{P}_{i, k+1}^{-1} & * & * \\
0 & -\mathcal{P}_{i, k+1}^{-1} & * \\
\bar{\beta}_{k} \mathcal{M}_{k}^{T} & \sqrt{\varphi_{k}} \mathcal{M}_{k}^{T} & -\frac{\mu_{i, k}}{2} I
\end{array}\right] . }
\end{aligned}
$$

Then, one arrives at (20) by performing congruent transformation $\operatorname{diag}\left\{I, I, I, I, \mathcal{P}_{i, k+1}, \mathcal{P}_{i, k+1}, I\right\}$ on (24). To this end, according to Theorem 1 , the filtering error system meets the required $H_{\infty}$-consensus performance criterion and the proof is thus complete.

Remark 3: To show the design flexibility of the proposed scheme, Theorem 2 is now compared with the corresponding results (without the RRP) in [11]. If the RRP is not implemented, it can be seen from Theorem 2 that only the second row and the second column of (20) include the information from neighbors of node $i$, i.e., $\Upsilon_{i j_{\hbar}, k}^{21}, \Upsilon_{i j_{\hbar}, k}^{22}$, and $\overline{\mathcal{K}}_{i j_{\hbar}, k}$. That is, only the second row and the second column of (20) can be locally adjusted to adapt to the changes of the neighboring nodes. Furthermore, it is observed from Theorem 2 that a distinctive feature of the proposed filter design algorithm is its flexible structure. Owing to the framework of the local design, Theorem 2 implies that there is no globally unknown information. As such, the filtering scheme proposed here can be executed distributedly by each node, thereby meriting the scalability of the proposed scheme.

Remark 4: Compared with the existing results, the distinctive features of the proposed algorithm include: 1) at each time step, only one neighboring node propagates its estimate for consensus thanks to the implementation of the RRP, and this can effectively avoid data collision and reduce communication burden; 2) a new performance index is proposed to characterize the noise attenuation level of filtering errors against external disturbances; and 3) the designed algorithm achieves the desired scalability and possesses high flexibility to the dynamical changes of the network topology. This investigation represents the first of the few attempts to develop local design methods for the distributed $H_{\infty}$-consensus filtering problem with RRP. 


\section{An ILlustrative ExAmple}

In this section, a simulation example is presented to illustrate the effectiveness of the proposed design scheme. Consider an $\mathrm{SN}$ with topology represented by a digraph $\mathcal{G}=(\mathcal{V}, \mathcal{E}, \mathscr{A})$ having the set of nodes $\mathcal{V}=\{1,2,3,4,5\}$, and the set of edges $\mathcal{E}=\{(2,1),(3,2),(4,3),(5,4),(5,1),(2,4),(5,3)\}$, as shown in Fig. 1. Obviously, $p_{1}=$ $0, p_{2}=2, p_{3}=p_{4}=1, p_{5}=3$, and $q_{1}=q_{3}=q_{4}=2, q_{2}=1, q_{5}=0$. Clearly, node 2 can receive information from nodes 1 and 4 in turn; node 5 can receive information from nodes 1,3 and 4 in turn. With the RRP, for a certain time step $k$, if $\bmod (k-1,2)=0$, then node 2 can receive information from node 1 , otherwise from node 4; if $\bmod (k-1,3)=0$, then node 5 can receive information from node 1 , else if $\bmod (k-1,3)=1$, then node 5 can receive information from node 3 , otherwise from node 4 .

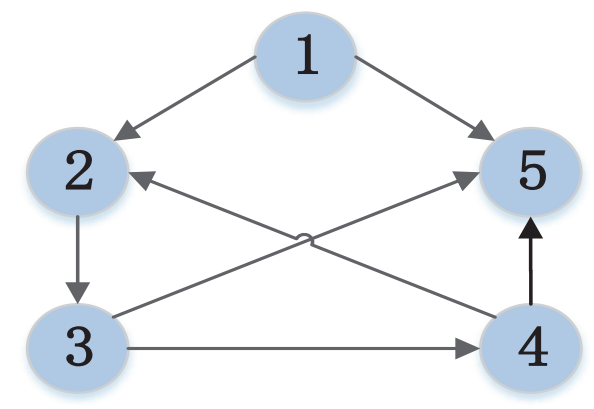

Fig. 1: Topology of a sensor network.

As described in [34], the parameter matrices for the continuous-time system are given as

$$
A_{c}=\left[\begin{array}{ccc}
-3.2 & 10 & 0 \\
1 & -1 & 1 \\
0 & -14.87 & 0
\end{array}\right], B_{c}=\left[\begin{array}{c}
-0.1246 \\
-0.4461 \\
0.3350
\end{array}\right]
$$

The nominal part of the above system represents the well-known Chua electronic circuit. By setting the sampling period $\Delta=0.2$, the parameter matrices of the corresponding discrete system are obtained as:

$$
A=\left[\begin{array}{ccc}
0.6472 & 1.2598 & 0.1492 \\
0.1260 & 0.7025 & 0.1737 \\
-0.2219 & -2.5834 & 0.7270
\end{array}\right], \quad B=\left[\begin{array}{c}
-0.0825 \\
-0.0732 \\
0.1845
\end{array}\right] \text {. }
$$

To reflect the frequently occurred parameter fluctuations, we allow certain time-varying variations and modify the parameter matrices as follows:

$$
A_{k}=\left[\begin{array}{ccc}
0.6472+0.1 \sin (k) & 1.2598 & 0.1492 \\
0.1260 & 0.7025 & 0.1737 \\
-0.2219 & -2.5834 & 0.7270
\end{array}\right], \quad B_{k}=\left[\begin{array}{c}
-0.0825+0.01 \sin (k) \\
-0.0732 \\
0.1845
\end{array}\right] .
$$

The other parameters are set as follows: $C_{1, k}=\left[\begin{array}{llll}0.0032 & -0.0047 & 0.001\end{array}\right], C_{2, k}=\left[\begin{array}{lll}0.01 & -0.1 & -0.013\end{array}\right]$, $C_{3, k}=\left[\begin{array}{ll}0.02-0.02 & 0.03\end{array}\right], C_{4, k}=\left[\begin{array}{ll}0.03-0.03 & 0.03\end{array}\right], C_{5, k}=\left[\begin{array}{lll}0.1 & 0 & 0\end{array}\right], D_{i, k}=0.025, \Delta A_{k}=\operatorname{diag}\{0.04 \sin (k), 0,0\}$, $\Delta B_{k}=\left[\begin{array}{lll}0.4 \sin (k) & 0 & 0\end{array}\right]^{T}, F_{k}=\sin (k), M_{k}=\left[\begin{array}{ll}0.2 & 0\end{array}\right]^{T}, N_{1 k}=\left[\begin{array}{ll}0.2 & 0\end{array}\right], N_{2 k}=0.2, z_{i, \max }=0.3, H_{11}=0.95, H_{21}=$ $0.8, H_{31}=0.75, H_{41}=0.65, H_{51}=0.7, H_{12}=1.65, H_{22}=1.5, H_{32}=1.45, H_{42}=1.35, H_{52}=1.4, n=51$, 
$\bar{\beta}_{k} \equiv 0.9, \gamma=0.3, \alpha_{i, k} \equiv 0.9, \mathcal{P}_{i, 0}=\operatorname{diag}\{I, 5 I\}, R_{i, k} \equiv 0.5 I, Q_{i, k} \equiv 0.5 I, U_{i}=100 \mathcal{P}_{i, 0}$, and $\mathcal{T}_{i, k} \equiv \operatorname{diag}\{1,1\}$. The disturbances are set as $w_{k}=0.1 \cos (5 k)$ and $\xi_{i, k}=0.1 \cos (5 k)$. By resorting to the YALMIP toolbox in MATLAB, all the desired filter gains are recursively computed based on Theorem 2.

The simulation results are presented in Figs. 2-6, where Fig. 2 plots the norms of the disagreement functions, Fig. 3 depicts the norms of the filtering errors, and Figs. 3-5 show the elements of $x_{k}$ and their estimations. The $j$-th element of $x_{k}$ and its estimation from node $i$ are denoted as $x_{k}^{(j)}$ and $\hat{x}_{i, k}^{(j)}(j=1,2,3 ; i=1, \cdots, 5)$, respectively. From the simulation results, it can be observed that the proposed distributed filtering algorithm is indeed effective.

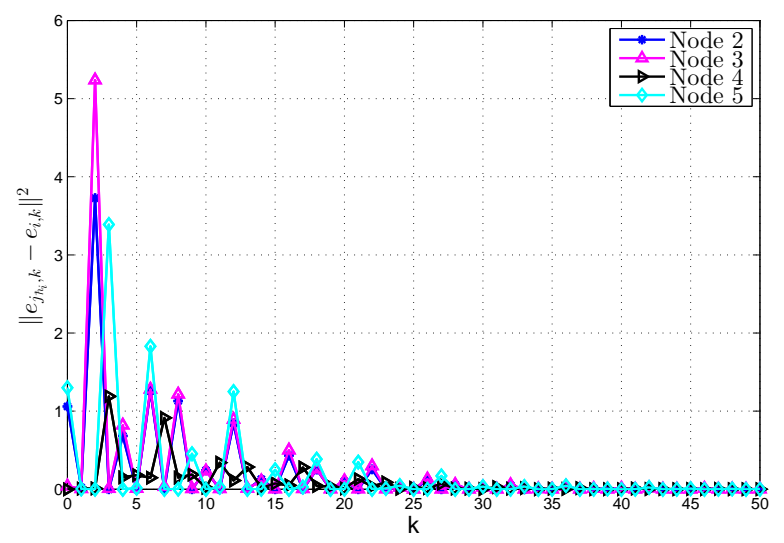

Fig. 2: The norms of disagreement functions.

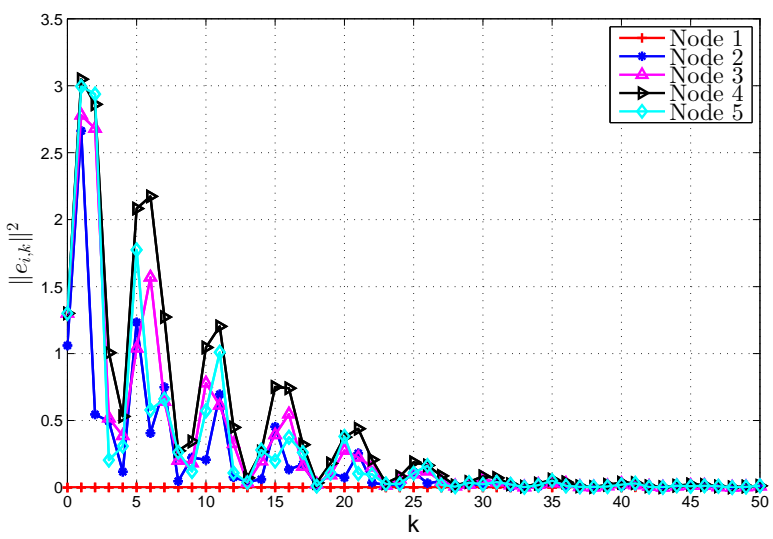

Fig. 3: The norms of filtering errors.

\section{CONCLUSION}

In this paper, the distributed $H_{\infty}$-consensus filtering problem has been investigated for a class of discrete timevarying systems subject to randomly occurring norm-bounded uncertainties and sensor saturations with the RRP over sensor networks. Typical norm-bounded uncertainties have been considered to enter into the network in a random manner. Sensor saturations are involved, reflecting the limited measurement capacity of nodes in SNs. In order to reduce the usage of network resources and sensor energy, the RRP has been employed to allow every node to receive information from only one neighboring node at each time step. Then, to establish the vector dissipativity 


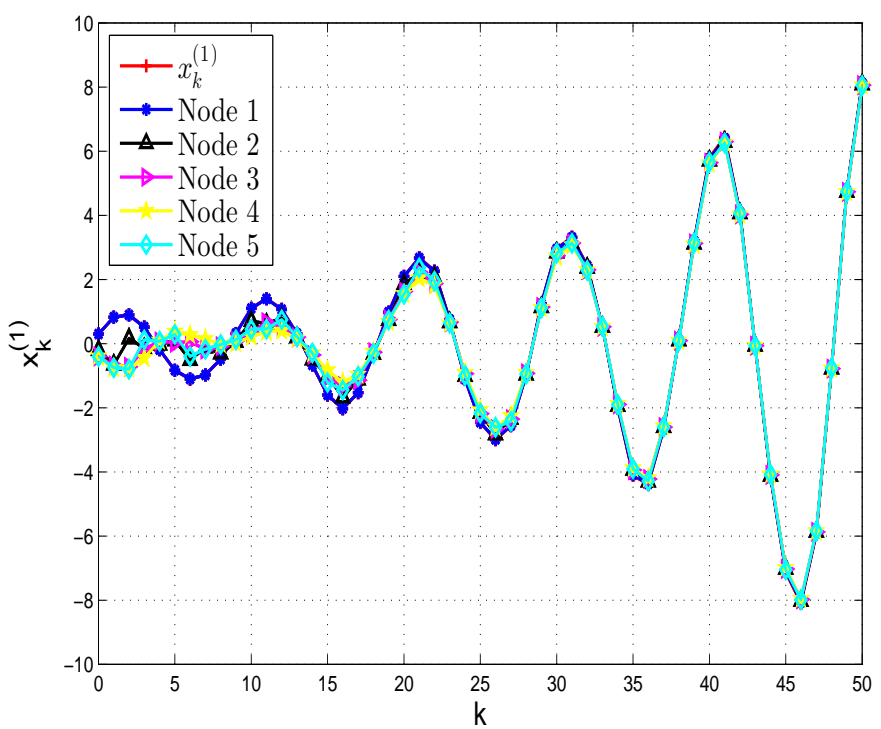

Fig. 4: $x_{k}^{(1)}$ and its estimations.

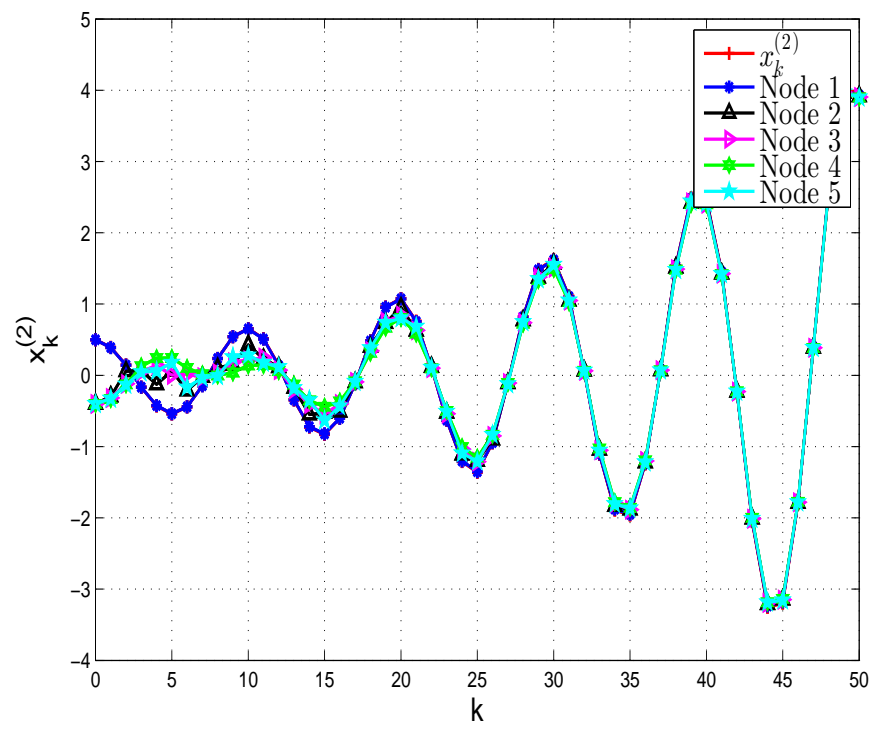

Fig. 5: $x_{k}^{(2)}$ and its estimations.

of the filtering error dynamics, the supply rate functions have been chosen according to the desired performance index. To that end, the vector dissipation inequality has been transformed to the desired $H_{\infty}$-consensus performance index utilizing some properties of the dissipation matrix. Sufficient conditions have been established for the filtering error dynamics to achieve pre-specified disturbance attenuation in the $H_{\infty}$ measure. Finally, a simulation example has been given to illustrate the effectiveness of the proposed distributed filtering scheme. In the future, these main results will be extended to more complicated systems with more comprehensive performance indices, similarly to the studies in e.g. [1], [8], [18], [20], [32], [42], [45], [46]. 


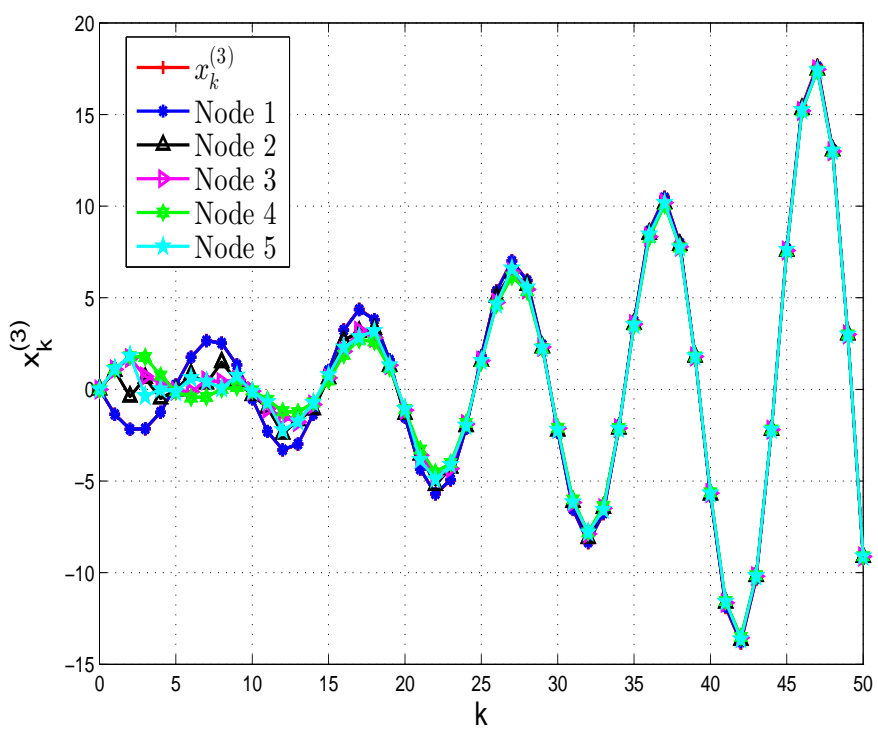

Fig. 6: $x_{k}^{(3)}$ and its estimations.

\section{REFERENCES}

[1] M. V. Basin, A. G. Loukianov, M. Hernandez-Gonzalez, Joint state and parameter estimation for uncertain stochastic nonlinear polynomial systems, International Journal of Systems Science, Vol. 44, No. 7, pp. 1200-1208, 2013.

[2] R. Caballero-Aguila, A. Hermoso-Carazo, J. Linares-Perez, Distributed fusion filters from uncertain measured outputs in sensor networks with random packet losses, Information Fusion, Vol. 34, pp. 70-79, 2017.

[3] B. Chen, G. Hu, D. W. C. Ho and L. Yu. Distributed Kalman filtering for time-varying discrete sequential systems, Automatica, Vol. 99, pp. 228-236, 2019.

[4] Y. Chen, Z. Wang, Y. Yuan and P. Date, Distributed $H_{\infty}$ filtering for switched stochastic delayed systems over sensor networks with fading measurements, IEEE Transactions on Cybernetics, Vol. 50, No. 1, pp. 2-14, 2020.

[5] D. Ciuonzo, A. De Maio and D. Orlando, On the statistical invariance for adaptive radar detection in partially homogeneous disturbance plus structured interference, IEEE Transactions on Signal Processing, Vol. 65, No. 5, pp. 1222-1234, 2017.

[6] M. C. F. Donkers, W. P. M. H. Heemels, N. van de Wouw and L. Hetel, Stability analysis of networked control systems using a switched linear systems approach, IEEE Transactions on Automatic Control, Vol. 56, No. 9, pp. 2101-2115, 2011.

[7] D. Ding, Z. Wang and Q. Han, A scalable algorithm for event-triggered state estimation with unknown parameters and switching topologies over sensor networks, IEEE Transactions on Cybernetics, Vol. 50, No. 9, pp. 4087-4097, 2020.

[8] H. Dong, N. Hou and Z. Wang, Fault estimation for complex networks with randomly varying topologies and stochastic inner couplings, Automatica, Vol. 112, Art. no. 108734, 2020.

[9] M. J. Garcia-Ligero, A. Hermoso-Carazo and J. Linares-Perez, Estimation from a multisensor environment for systems with multiple packet dropouts and correlated measurement noises, Applied Mathematical Modelling, Vol. 45, pp. 802-812, 2017.

[10] X. Ge and Q.-L. Han, Distributed sampled-data asynchronous $H_{\infty}$ filtering of Markovian jump linear systems over sensor networks, Signal Processing, Vol. 127, pp. 86-99, 2016.

[11] F. Han, G. Wei, D. Ding and Y. Song, Local condition-based consensus filtering with stochastic nonlinearities and multiple missing measurements, IEEE Transactions on Automatic Control, Vol. 62, No. 9, pp. 4784-4790, 2017.

[12] F. Han, H. Dong, Z. Wang and G. Li, Local design of distributed $H_{\infty}$-consensus filtering over sensor networks under multiplicative noises and deception attacks, International Journal Robust and Nonlinear Control, Vol. 29, No. 8, pp. 2296-2314, 2019.

[13] F. Han, D. Ding, F. Yang and W. Gao, Distributed resilient estimation for nonlinear delay systems with stochastic perturbations, International Journal of Roubust Nonlinear Control, Vol. 30, pp. 843-863, 2020.

[14] J. Hu, Z. Wang, J. Liang and H. Dong, Event-triggered distributed state estimation with randomly occurring uncertainties and nonlinearities over sensor networks: A delay-fractioning approach, Journal of the Franklin Institute, Vol. 352, No. 9, pp. 3750-3763, 2015 . 
[15] J. Hu, Z. Wang, G.-P. Liu, H. Zhang and R. Navaratne, A prediction-based approach to distributed filtering with missing measurements and communication delays through sensor networks, IEEE Transactions on Systems, Man, and Cybernetics-Systems, in press, DOI: 10.1109/TSMC.2020.2966977.

[16] W. Haddad, V. Chellabolna, and S. Nersesov, Vector dissipativity theory and stability of feedback interconnections for large-scale non-linear dynamical systems, International Journal of Control, Vol. 77, No. 10, pp. 907-919, 2004.

[17] J. Li, Z. Wang, H. Dong and F. Han, Delay-distribution-dependent state estimation for neural networks under stochastic communication protocol with uncertain transition probabilities, Neural Networks, vol. 130, pp. 143-151, 2020.

[18] J. Li, Z. Wang, H. Dong and G. Ghinea, Outlier-resistant remote state estimation for recurrent neural networks with mixed time-delays, IEEE Transactions on Neural Networks and Learning Systems, in press, DOI: 10.1109/TNNLS.2020.2991151.

[19] Q. Li, B. Shen, Z. Wang and W. Sheng, Recursive distributed filtering over sensor networks on Gilbert-Elliott channels: A dynamic event-triggered approach, Automatica, Vol. 113, Art. no. 108681, 2020.

[20] W. Li, C. Meng, Y. Jia and J. Du, Recursive filtering for complex networks using non-linearly coupled UKF, IET Control Theory \& Applications, Vol. 12, No. 4, pp. 549-555, 2018.

[21] J. Liang, B. Shen, H. Dong and J. Lam, Robust distributed state estimation for sensor networks with multiple stochastic communication delays, International Journal of Systems Science, Vol. 42, No. 9, pp. 1459-1471, 2011.

[22] S. Liu, Z. Wang, G. Wei, and M. Li, Distributed set-membership filtering for multi-rate systems under the Round-Robin scheduling over sensor networks, IEEE Transactions on Cybernetics, vol. 50, No. 5, pp. 1910-1920, 2020.

[23] K. Liu, E. Fridman and L. Hetel, Stability and $L_{2}$-gain analysis of networked control systems under round-robin scheduling: A timedelay approach, Systems \& Control Letters, Vol. 61, No. 5, pp. 666-675, 2012.

[24] Q. Lu, Q.-L. Han, B. Zhang, D. Liu and S. Liu, Cooperative control of mobile sensor networks for environmental monitoring: an event-triggered finite-time control scheme, IEEE Transactions on Cybernetics, Vol. 47, No. 12, pp. 4134-4147, 2017.

[25] L. Ma, Z. Wang, C. Cai and F. E. Alsaadi, A dynamic event-triggered approach to $H_{\infty}$ control for discrete-time singularly perturbed systems with time-delays and sensor saturations, IEEE Transactions on Systems, Man, and Cybernetics-Systems, in press, DOI: 10.1109/TSMC.2019.2958529.

[26] L. Ma, Z. Wang, Hak-Keung Lam and N. Kyriakoulis, Distributed event-based set-membership filtering for a class of nonlinear systems with sensor saturations over sensor networks, IEEE Transactions on Cybernetics, Vol. 47, No. 11, pp. 3772-3783, 2017.

[27] J. M. Pak, C. K. Ahn, P. Shi, Y. S. Shmaliy and M. T. Lim, Distributed hybrid particle/FIR filtering for mitigating NLOS effects in TOA-based localization using wireless sensor networks, IEEE Transactions on Industrial Electronics, Vol. 64, No. 6, pp. 5182-5191, 2017.

[28] B. Shen, Z. Wang, D. Wang and H. Liu, Distributed state-saturated recursive filtering over sensor networks under Round-Robin protocol, IEEE Transactions on Cybernetics, Vol. 50, No. 8, pp. 3605-3615, 2020.

[29] Y. Shen, Z. Wang, B. Shen and F. E. Alsaadi, $H_{\infty}$ filtering for multi-rate multi-sensor systems with randomly occurring sensor saturations under the p-persistent CSMA protocol, IET Control Theory and Applications, vol. 14, no. 10, pp. 1255-1265, Jul. 2020.

[30] S. Sun, F. Peng and H. Lin, Distributed asynchronous fusion estimator for stochastic uncertain systems with multiple sensors of different fading measurement rates, IEEE Transactions on Signal Processing, Vol. 66, No. 3, pp. 641-653, 2018.

[31] S. Sun, H. Lin, J. Ma and X. Liu, Multi-sensor distributed fusion estimation with applications in networked systems: A review paper, Information Fusion, Vol. 38, pp. 122-134, 2017.

[32] M. Tong, W. Lin, X. Huo and Z. Jin, A model-free fuzzy adaptive trajectory tracking control algorithm based on dynamic surface control, International Journal of Advanced Robotic Systems, Vol. 17, No. 1, Art. no. 1729881419894417, 2020.

[33] V. Ugrinovskii, Distributed robust estimation over random switching networks using $H_{\infty}$ consensus, Automatica, Vol. 49, No. 1, pp. 160-168, 2013.

[34] V. Ugrinovskii and E. Fridman, A round-robin type protocol for distributed estimation with $H_{\infty}$ consensus, Systems \& Control Letters, Vol. 69, pp. 103-110, 2014.

[35] X. Wan, Z. Wang, M. Wu and X. Liu, $H_{\infty}$ state estimation for discrete-time nonlinear singularly perturbed complex networks under the Round-Robin protocol, IEEE Transactions on Neural Networks and Learning Systems, Vol. 30, No. 2, pp. 415-426, 2019.

[36] H-D. Wang and H-N. Wu, Design of finite dimensional robust $H_{\infty}$ distributed consensus filters for dissipative PDE systems with sensor networks, International Journal of Robust and Nonlinear Control, Vol. 25, No. 10, pp. 1454-1471, 2015.

[37] Y. Xu, R. Lu, P. Shi, H. Li and S. Xie, Finite-time distributed state estimation over sensor networks with Round-Robin protocol and fading channels, IEEE Transactions on Cybernetics, Vol. 48, No. 1, pp. 336-345, 2018.

[38] W. Yang, C. Yang, H. Shi, L. Shi and G. Chen, Stochastic link activation for distributed filtering under sensor power constraint, Automatica, Vol. 75, pp. 109-118, 2017. 
[39] H. Yan, Q. Yang, H. Zhang, F. Yang and X. Zhan. Distributed $H_{\infty}$ state estimation for a class of filtering networks with time-varying switching topologies and packet losses, IEEE Transactions on System, Man, and Cybernetics: Systems, Vol. 48, No. 12, pp. 2047-2057, 2018.

[40] W. Yu, G. Chen, Z. Wang and W. Yang, Distributed consensus filtering in sensor networks, IEEE Transactions on Systems, Man and Cybernetics-Part B, Vol. 39, No. 6, pp. 1568-1577, 2009.

[41] D. Zhang, Z. Xu, H. R. Karimi and Q. Wang, Distributed filtering for switched linear systems with sensor networks in presence of packet dropouts and quantization, IEEE Transactions on Circuits and Systems-Part I, Vol. 64, No. 10, pp. $2783-2796,2017$.

[42] X.-M. Zhang, Q.-L. Han, X. Ge, D. Ding, L. Ding, D. Yue and C. Peng, Networked control systems: A survey of trends and techniques, IEEE/CAA Journal of Automatica Sinica, Vol. 7, No. 1, pp. 1-17, 2020.

[43] D. Zhao, Z. Wang, Y. Chen and G. Wei, Proportional-integral observer design for multi-delayed sensor-saturated recurrent neural networks: A dynamic event-triggered protocol, IEEE Transactions on Cybernetics, in press, DOI: 10.1109/TCYB.2020.2969377

[44] Y. Zhao, X. He and D. Zhou, Distributed filtering for time-varying networked systems with sensor gain degradation and energy constraint: a centralized finite-time communication protocol scheme, Science China: Information Sciences, Vol. 61, No. 9, Art. no. $092208,2018$.

[45] L. Zou, Z. Wang, Q.-L. Han and D. H. Zhou, Recursive filtering for time-varying systems with random access protocol, IEEE Transactions on Automatic Control, Vol. 64, No. 2, pp. 720-727, 2019.

[46] L. Zou, Z. Wang, J. Hu and D. H. Zhou, Moving horizon estimation with unknown inputs under dynamic quantization effects, IEEE Transactions on Automatic Control, in press, DOI: 10.1109/TAC.2020.2968975.

[47] L. Zou, Z. Wang, Q.-L. Han and D. Zhou, Moving horizon estimation for networked time-delay systems under Round-Robin protocol, IEEE Transactions on Automatic Control, Vol. 64, No. 12, pp. 5191-5198, 2019.

[48] L. Zou, Z. Wang, Q.-L. Han and D. H. Zhou, Full information estimation for time-varying systems subject to Round-Robin scheduling: A recursive filter approach, IEEE Transactions on Systems, Man, and Cybernetics-Systems, in press, DOI: 10.1109/TSMC.2019.2907620. 